\title{
MUON SPIN ROTATION STUDIES
} OF THE VORTEX MATTER IN THE HIGH- $T_{\mathrm{c}}$ SUPERCONDUCTOR

\section{$\mathrm{Bi}_{2} \mathrm{Sr}_{2} \mathrm{CaCu}_{2} \mathrm{O}_{8+\delta}$}

T. Blasius ${ }^{a}$, Ch. Niedermayer ${ }^{a}$, J.L. Tallon ${ }^{b}$, D.M. Pooke ${ }^{b}$, A. GolniK ${ }^{c}$, D.R. NoAKes ${ }^{d}$, C.E. StronaCH ${ }^{d}$, E.J. ANSALDo ${ }^{e}$, R.W. $\operatorname{HENN}^{f}$, C.T. $\operatorname{LIN}^{f}$ AND C. BERNHARD $f$

${ }^{a}$ Universität Konstanz, Fakultät für Physik, 78457 Konstanz, Germany

${ }^{b}$ The New Zealand Institute for Industrial Research

P.O. Box 31310, Lower Hutt, New Zealand

'Institute of Experimental Physics, Warsaw University, 00-681 Warsaw, Poland

${ }^{d}$ Virginia State University, Petersburg, Virginia 23806, USA

' University of Saskatchewan, Saskatoon, Saskatchewan, Canada S7N 0W0

${ }^{f}$ Max-Planck-Institut für Festkörperforschung, 70569 Stuttgart, Germany

We report muon spin rotation studies of the magnetic field distribution $n(B)$ in the vortex state of the high-temperature superconductor $\mathrm{Bi}_{2} \mathrm{Sr}_{2} \mathrm{CaCu}_{2} \mathrm{O}_{8+\delta}$. From our data on three sets of overdoped, nearly optimized and underdoped single crystals we obtain evidence for a two-stage melting process of the vortex matter under equilibrium conditions. In the irreversible regime well below the irreversibility line we observe a transition in the vortex state which we associate with an intra-planar melting transition. The resulting flux-line liquid phase persists over a sizeable temperature interval before the inter-planar decoupling of the individual vortex lines takes place in a second step at the irreversibility line, resulting in a pancake liquid phase.

PACS numbers: $74.60 . \mathrm{Ge}, 74.25 . \mathrm{Dw}, 74.72 . \mathrm{Hs}, 76.75 .+\mathrm{i}$

The vortex matter in cuprate high- $T_{\mathrm{c}}$ superconductors exhibits a complex $(H, T)$-phase diagram as a function of magnetic field, temperature and doping [1]. The short superconducting (SC) coherence length, the high transition temperatures and the strongly anisotropic layered structure favor thermal fluctuations which dominate the vortex state well below the upper critical field $H_{\mathrm{c} 2}(T)$. In contrast to conventional type-II superconductors, a melting transition of the flux-line lattice (FLL) was proposed [2] and confirmed experimentally [3]. Nevertheless, the details of this melting transition are not yet fully understood. 
When the external magnetic field is applied perpendicular to the $\mathrm{CuO}_{2}$ planes along the $c$-axis direction $(H \| c$ ) the flux-lines can be viewed as stacks of pancake vortices that reside within the superconducting $\mathrm{CuO}_{2}$ planes and are weakly coupled across the insulating spacer layers mainly via Josephson tunneling [4]. Both the intra-planar vortex order and the inter-planar coupling between the pancake vortices that form a flux-line can be overcome by thermal fluctuations. In principle, these processes may occur independently and at different temperatures, depending on the anisotropy and the condensation energy or the superfluid density which determine the $c$-axis coupling strength and the in-plane vortex-vortex interaction, respectively.

Based on these considerations it has been proposed that the FLL may undergo a two-stage melting transition where the intra-planar melting associated with the transition from a flux-line lattice to a flux-line liquid occurs at a lower temperature than the decoupling of the individual flux-lines which results in a pancake liquid [5]. Recently, experimental evidence for this two-stage melting scenario has been obtained from $c$-axis transport measurements [6] and vortex penetration through surface barriers in the presence of transport current [7] on strongly anisotropic $\mathrm{Bi}_{2} \mathrm{Sr}_{2} \mathrm{CaCu}_{2} \mathrm{O}_{8+\delta}$ (Bi-2212). These experiments, however, probe the vortex state under non-equilibrium conditions. The equilibrium vortex structure can be studied with techniques like small-angle neutron scattering (SANS) and transverse-field muon spin rotation ( $\mathrm{TF}-\mu \mathrm{SR}$ ). Both techniques have provided valuable information on the vortex state, in particular, on the vortex matter transition at the irreversibility line (IL) and the so-called "dimensional crossover" from a $3 \mathrm{D}$ to a $2 \mathrm{D}$ vortex structure that occurs with increasing magnetic field [8-10].

Here we present transverse-field muon spin rotation data on three sets of overdoped $\left(T_{\mathrm{c}}=64 \mathrm{~K}\right)$, nearly optimized $\left(T_{\mathrm{c}}=90 \mathrm{~K}\right)$, and underdoped $\left(T_{\mathrm{c}}=\right.$ $77 \mathrm{~K}$ ) Bi-2212 single crystals which to our best knowledge provide the first evidence that a two-stage melting transition of the vortex matter occurs under equilibrium conditions [11]. In addition, we compare our $\mathrm{TF}-\mu \mathrm{SR}$ results for the overdoped and underdoped single crystals with those from DC-magnetization measurements obtained on the same samples with a vibrating-sample magnetometer (VSM) [10].

The two sets of underdoped and overdoped crystals were grown by a floating-zone technique [12]. The oxygen content has been achieved by annealing in a gas stream of $0.02 \%$ oxygen in nitrogen at $765^{\circ} \mathrm{C}$ followed by rapid quenching for the underdoped set and by slow cooling from $550^{\circ} \mathrm{C}$ to $350^{\circ} \mathrm{C}$ in 60 atm oxygen for the overdoped set. The set of nearly optimized crystals was grown by a flux method with a cooling rate of $0.3 \mathrm{~K} / \mathrm{h}$ [13]. The as-grown crystals exhibit a very sharp superconducting transition at $T_{\mathrm{c}}=90 \mathrm{~K}$.

The TF- $\mu \mathrm{SR}$ experiments have been performed at the $\pi \mathrm{M} 3$ muon beam line at the Paul-Scherrer-Institut in Villigen, Switzerland and the M-15 muon beam line at TRIUMF in Vancouver, Canada. In TF- $\mu$ SR experiments $100 \%$ spin-polarized positive muons are implanted into the bulk of the crystal. An external magnetic field $H_{\text {ext }}$ is applied perpendicular to the initial polarization of the muon spins (along the crystallographic $c$-axis). The muons thermalize rapidly without any significant loss in polarization and come to rest about 100-200 $\mu \mathrm{m}$ below the surface at interstitial locations, $r$, which are randomly distributed on the length 
scale of the magnetic penetration depth $\lambda$. Their spins start to precess in the local magnetic field $B_{\text {int }}(r)$ with the Larmor frequency $\omega_{\mu}=\gamma_{\mu} B_{\text {int }}(r)$, where $\gamma_{\mu}=851.4 \mathrm{MHz} / \mathrm{T}$ is the gyromagnetic ratio of the muon. The time evolution of the so-called muon spin polarization function $P(t)$ is then measured by monitoring the decay positrons which are preferentially emitted along the muon spin direction at the instant of decay (half lifetime $2.2 \mu \mathrm{s}$ ). The probability distribution of the local magnetic field $n(B)$, the so-called " $\mu$ SR-lineshape", is extracted from $P(t)$ either via fast Fourier transform (FFT) techniques, real part of the FFT [14], or maximum entropy (ME) technique [15] and contains detailed information on the vortex structure. A schematic diagram of such a TF- $\mu \mathrm{SR}$ experiment is shown in Fig. 1. A representative asymmetry spectrum in time space and the corresponding
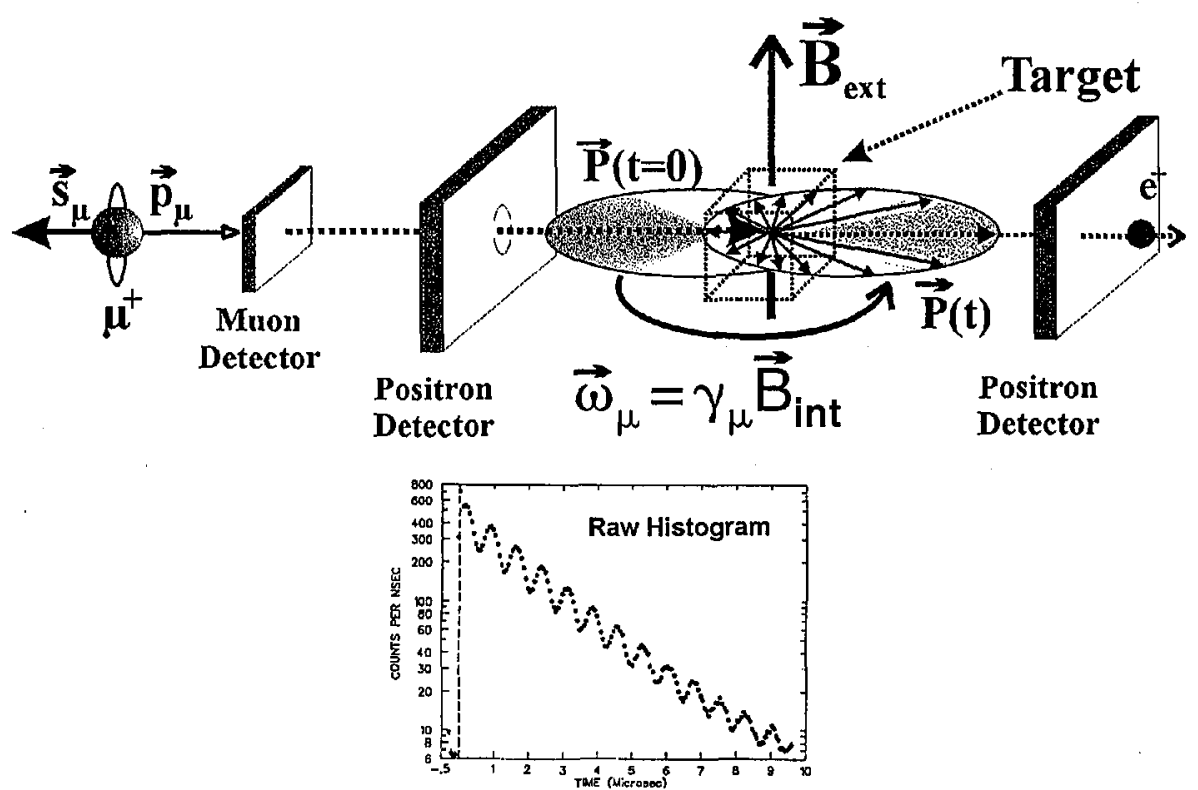

Fig. 1. Schematic diagram showing the principle of a TF- $\mu \mathrm{SR}$ experiment: the muon beam enters from the left with its polarization antiparallel to its momentum. A magnetic field is applied to the sample, transverse to the muon spins. The internal field, $B_{\text {int }}$, in the sample, where the muons come to rest, causes a precession of the muon spins with the Larmor frequency $\omega_{\mu}=\gamma_{\mu} B_{\text {int }}(r)$, where $\gamma_{\mu}=851.4 \mathrm{MHz} / \mathrm{T}$ is the gyromagnetic ratio of the muon. The incoming muon passes through a plastic scintillator (muon detector) and generates a start pulse for a time digital converter ("clock"). The clock is stopped by the detection of a decay positron in one of the positron counters surrounding the sample. For each such event the time interval is digitized and the corresponding bin in a discrete time histogram is incremented. The inset shows a "raw" time spectrum: the overall exponential decay reflects the muon lifetime and the precession of the muon spins is manifested in the superimposed oscillations as the muon polarization sweeps past the positron detector (for more details, see [16]). 

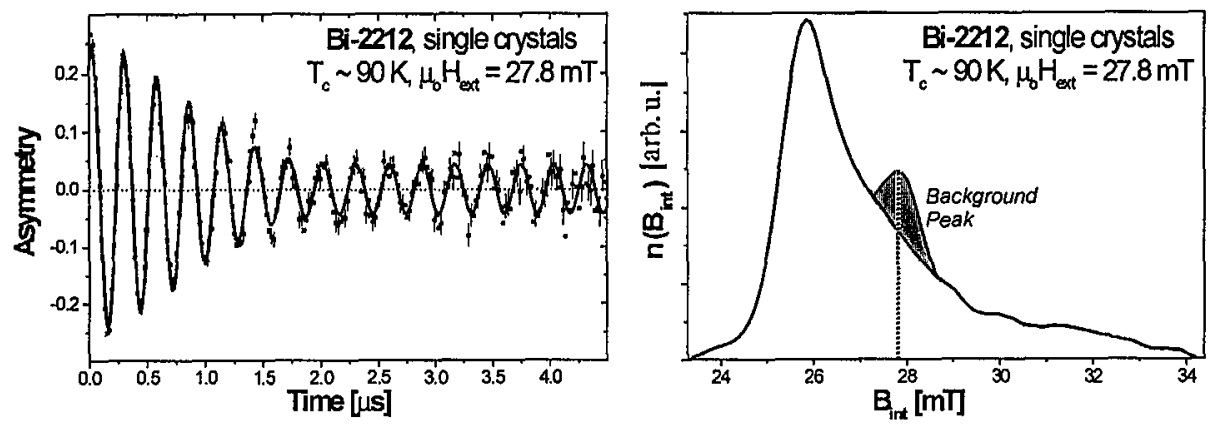

Fig. 2. (Left) TF- $\mu \mathrm{SR}$ asymmetry spectrum obtained from the raw time spectrum by subtracting any time-independent background and dividing out the exponential distribution of muon decay times by combination of pairs of positron detectors (for more details, see [16]). (Right) Corresponding internal field distribution extracted from the asymmetry spectrum via fast Fourier transformation. The so-called background peak is due to muons that do not stop in the sample and can be subtracted in the data analysis assuming a Gaussian distribution.

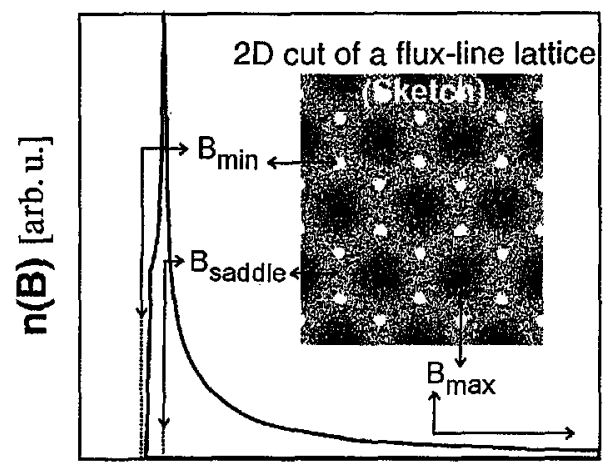

Internal Field [arb.u.]

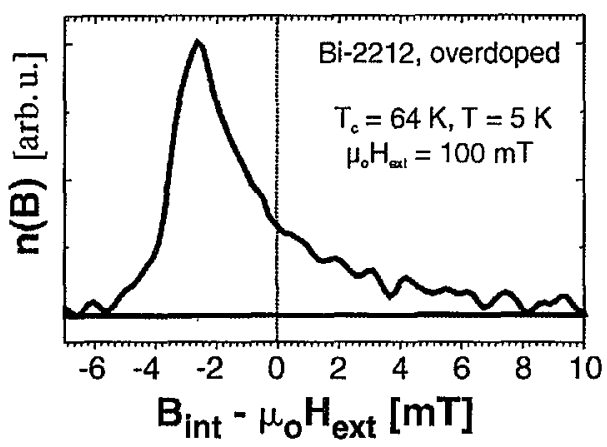

Fig. 3. (Left) Resulting internal field distribution due to a static and perfect 3D Abrikosov flux line lattice. (Right) Internal field distribution as measured in a field cooled TF- $\mu \mathrm{SR}$ experiment on an overdoped Bi-2212 single crystal.

probability distribution of fields, $n(B)$ (received from the distribution in frequency space using $\left.\omega_{\mu}=\gamma_{\mu} B_{\text {int }}(r)\right)$ is shown in Fig. 2. For a more detailed description of the TF- $\mu \mathrm{SR}$ technique, see [16].

For a three-dimensional (3D) flux line lattice, $n(B)$ is strongly asymmetric [17] with a pronounced tail towards high fields due to muons that stop near the vortex cores, a cusp which corresponds to the field at the saddle point between adjacent vortices and a cutoff on the low field side corresponding to the field minimum at the point which is most remote from the vortex cores as shown in Fig. 3. The resulting asymmetry or "skewness" of the " $\mu$ SR-lineshape" can be characterized by calculating the second and third moment of the internal magnetic 
field distribution and defining the dimensionless parameter [9] $\alpha \equiv\left\langle\Delta B^{3}\right\rangle^{1 / 3}$ $\left\langle\Delta B^{2}\right\rangle^{1 / 2}=\left[\int(B-\langle B\rangle)^{3} n(B) \mathrm{d} B\right]^{1 / 3} /\left[\int(B-\langle B\rangle)^{2} n(B) \mathrm{d} B\right]^{1 / 2}$, where $\left\langle\Delta B^{n}\right\rangle$ is the $n$-th central moment and $\langle B\rangle=\int B n(B) \mathrm{d} B$ is the expectation value of the internal field distribution. A value of $\alpha \approx 1$ is typical of the " $\mu$ SR-lineshape" due to a static 3D FLL [9-11]. A reduced value of $1>\alpha>0$ either indicates a disorder of the static vortex structure or else vortex dynamics in excess of the typical $\mu \mathrm{SR}$ time scale of $\tau \leq 10^{-6} \mathrm{~s}$ [9-11]. From the $\mu \mathrm{SR}$ experiments alone one cannot distinguish between these two possibilities. Further information regarding the static or dynamic nature of the vortex state, however, can be obtained from DC-magnetization measurements.

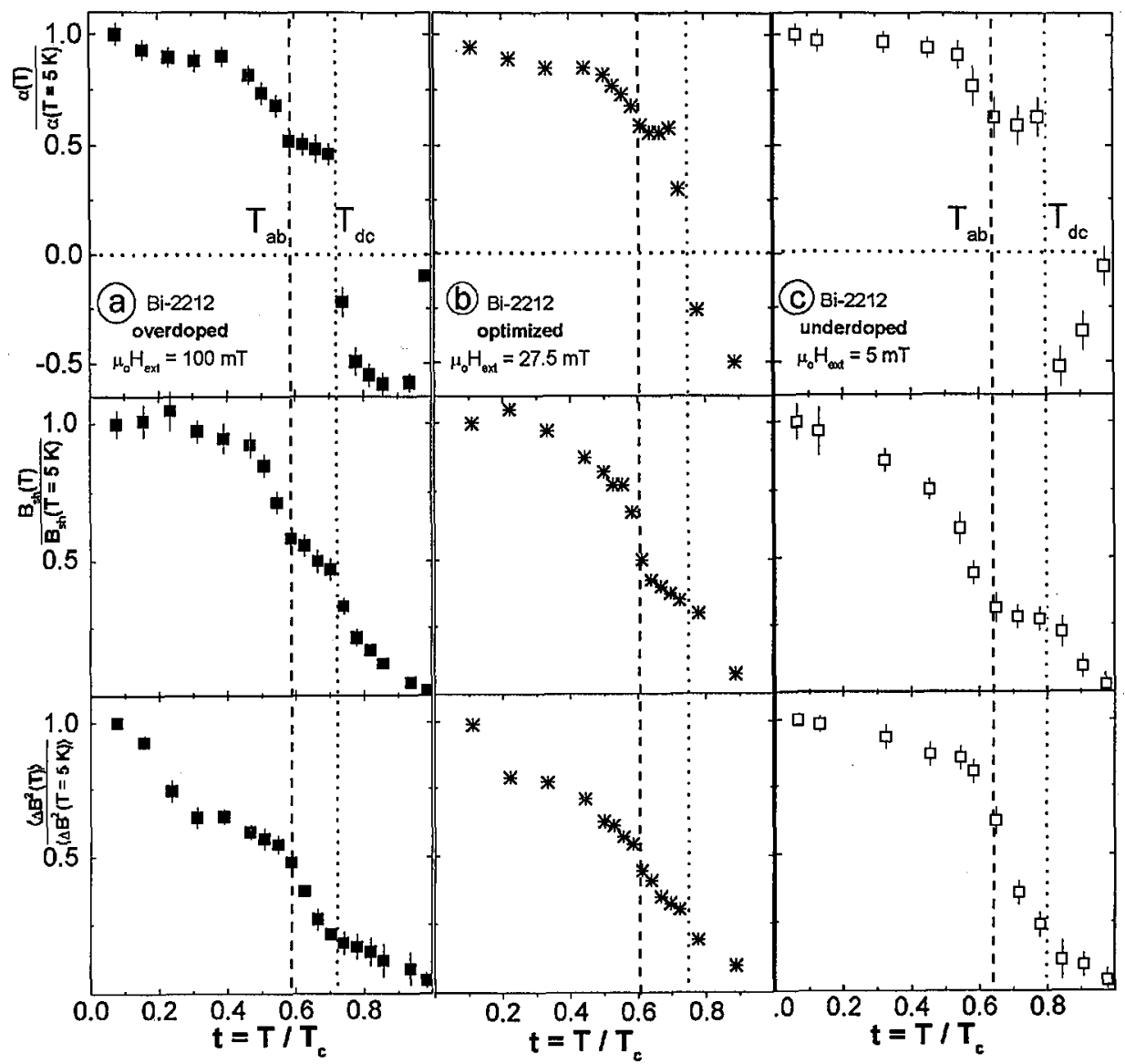

Fig. 4. Data from field-cooled TF- $\mu \mathrm{SR}$ experiments on sets of Bi-2212 single crystals (overdoped, $T_{\mathrm{c}}=64 \mathrm{~K}$, nearly optimized $T_{\mathrm{c}}=90 \mathrm{~K}$, and underdoped $T_{\mathrm{c}}=77 \mathrm{~K}$ in an applied field of $\mu_{0} H_{\text {ext }}=100 \mathrm{mT}$ (a), $27.5 \mathrm{mT}$ (b), and $5 \mathrm{mT}$ (c) respectively). Temperature dependence of the "skewness" $\alpha$ (top), the cusp-shift (middle), and the second moment (bottom) of $n(B) . T_{\mathrm{ab}}$ and $T_{\mathrm{dc}}$ denote the temperature for the intra-planar melting and the inter-planar decoupling, respectively [11]. 
With increasing temperature a 3D FLL will be subject to thermal fluctuations and $\alpha$ will be continuously reduced from $\alpha \approx 1$ at low temperature towards rather small but still positive $\alpha$-values at elevated temperatures. In contrast, $\alpha$ has been shown to undergo a sudden and discontinuous change from positive to negative values at the irreversibility line $[9,18]$. This behavior has been attributed to the melting transition of the vortex structure $[9,18]$. Previous TF- $\mu$ SR experiments gave no indication that an additional transition (as a function of temperature for a constant applied field) of the vortex state occurs in the irreversible regime below the IL. The transition associated with the IL therefore has been ascribed to a one-stage melting of the vortex lattice where the intra-planar melting and inter-planar decoupling occur simultaneously at the temperature $T_{\mathrm{m}}[9,18]$.

In the following we present new TF- $\mu \mathrm{SR}$ data which indicate that a second transition occurs in the irreversible regime well below the IL. Figure 4 summarizes the TF- $\mu$ SR results for $\alpha$, the shift of the cusp-field with respect to the external field (cusp-shift, $B_{\text {sh }}$ ) and the second moment of the " $\mu$ SR-lineshape" $\left\langle\Delta B^{2}\right\rangle$. The data have been obtained by field cooling in an applied field of $100 \mathrm{mT}$ for the overdoped set, $27.5 \mathrm{mT}$ for the nearly optimized set, and $5 \mathrm{mT}$ for the underdoped set. In all cases the external field is well below the dimensional crossover field $H^{*}\left(H^{*} \approx 150 \mathrm{mT}\right.$ and $\approx 7.5 \mathrm{mT}$ for the overdoped and underdoped crystals [10] and $\approx 60 \mathrm{mT}$ for the optimized crystals) and the signature of a $3 \mathrm{D}$ vortex lattice, i.e. $\alpha \approx 1$, is observed at low temperature. In agreement with previous reports $[9,18]$ the " $\mu$ SR-lineshape" exhibits the most pronounced changes in the vicinity of the IL, where $\alpha$ exhibits a sudden drop and changes sign at $T_{\mathrm{dc}} \approx 46.5 \mathrm{~K}$ $\left(0.73 T_{\mathrm{c}}\right)$ for the overdoped, $T_{\mathrm{dc}} \approx 67.5 \mathrm{~K}\left(0.75 T_{\mathrm{c}}\right)$ for the nearly optimized, and $T_{\mathrm{dc}} \approx 61.5 \mathrm{~K}\left(0.80 T_{\mathrm{c}}\right)$ for the underdoped sample, respectively (Fig. 4, top panels). In addition, the $T$-dependence of the " $\mu$ SR-lineshape" exhibits a second pronounced anomaly where a significant change is evident for the "skewness", the cusp-shift and the second moment of the " $\mu$ SR-lineshape" at a temperature $T_{\mathrm{ab}}$ (Fig. 4) well below the IL. We argue that the observed transition may be related to the intra-planar melting of the vortex lattice (at $T_{\mathrm{ab}}$ ) to a flux-line liquid phase. This phase persists over a sizable temperature interval for $T_{\mathrm{ab}}<T<T_{\mathrm{dc}}$ before the individual flux-lines are decoupled at a significantly higher temperature $T_{\mathrm{dc}}$ at the IL.

We argue that the observed transition at $T_{\mathrm{ab}}$ in the irreversible state cannot be easily explained in terms of a crossover in the vortex dynamics, which in the relevant temperature range is very different for the underdoped/nearly optimized and the overdoped crystals. We have studied the pinning of the vortex lattice with the TF- $\mu$ SR technique by reducing the applied field after the field-cooling process. This is shown in Fig. 5 for a nearly optimized single crystal. For a rigidly pinned vortex state $n(B)$ will not follow the change of the applied field, whereas for a depinned vortex array $n(B)$ will adjust to the changes of $H_{\text {ext }}$. We observe for the overdoped single crystals the onset of vortex motion already at a temperature $T_{\mathrm{dp}} \approx 17 \mathrm{~K}\left(0.27 T_{\mathrm{c}}\right) \ll T_{\mathrm{ab}} \approx 37 \mathrm{~K}\left(0.58 T_{\mathrm{c}}\right)$ for an applied field of $100 \mathrm{mT}$ (field cooling) which had been reduced by $10 \mathrm{mT}$ at $T=5 \mathrm{~K}$. The signature of the vortex motion is evident also from the pronounced decrease in $\left\langle\Delta B^{2}\right\rangle$ (Fig. 4a, bottom panel) and the modest reduction of $\alpha$ (Fig. 4a, top panel) towards $T_{\mathrm{dp}}$ 


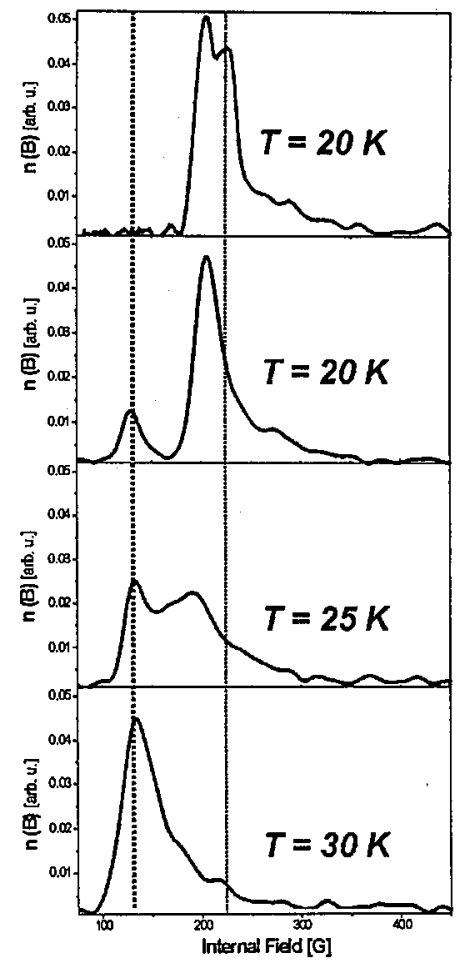

Fig. 5. Internal field distributions received from pinning experiments on a nearly optimized set of Bi-2212 single crystals. For details see text.

in the $T$-scans. In contrast, for the underdoped set we obtain no evidence that significant vortex motion sets in below $T_{\mathrm{ab}}$, neither from the pinning experiments nor from the $T$-dependence of $\alpha$ and $\left\langle\Delta B^{2}\right\rangle$. Here the sudden onset of vortex motion coincides with the transition at $T_{\mathrm{ab}}$. The profound difference in the vortex dynamics of the two sets of underdoped and overdoped crystals makes it rather unlikely that the significant and analogous changes of the " $\mu$ SR-lineshape" at $T_{\mathrm{ab}}$ arise simply from a crossover in the vortex dynamics.

Possibly even more instructive is the $T$-dependence of the " $\mu$ SR-lineshape" which we obtain for applied magnetic fields $H>H^{*}$. Here the low-temperature vortex-state is a quasi-2D lattice of pancake vortices [9-11]. Roughly speaking, the phase coherence across the blocking layers is destroyed once the pancake vortices in adjacent $\mathrm{CuO}_{2}$ blocks are randomly displaced on a length scale comparable to their a verage spacing $a_{0}=\left(\Phi_{0} / B\right)^{1 / 2}$ within the $\mathrm{CuO}_{2}$ planes, where $\Phi_{0}$ is the flux quantum. Such displacements, by thermal fluctuations or random pinning, are opposed by the cost in the Josephson energy. The Josephson length $\lambda_{\mathrm{J}}=\gamma s$ (anisotropy $\gamma=\lambda_{c} / \lambda_{\mathrm{ab}}$ and $s$ is the $\mathrm{CuO}_{2}$ block distance, Fig. 6) defines a characteristic length scale over which pinning and fluctuations are effective. Assuming $a_{0}=\lambda_{\mathbf{J}}$ as the criterion for the decoupling of the pancake vortices in different $\mathrm{CuO}_{2}$ blocks results in $B^{*}=\Phi_{0} /(\gamma s)^{2}$. Furthermore, it has been suggested that 


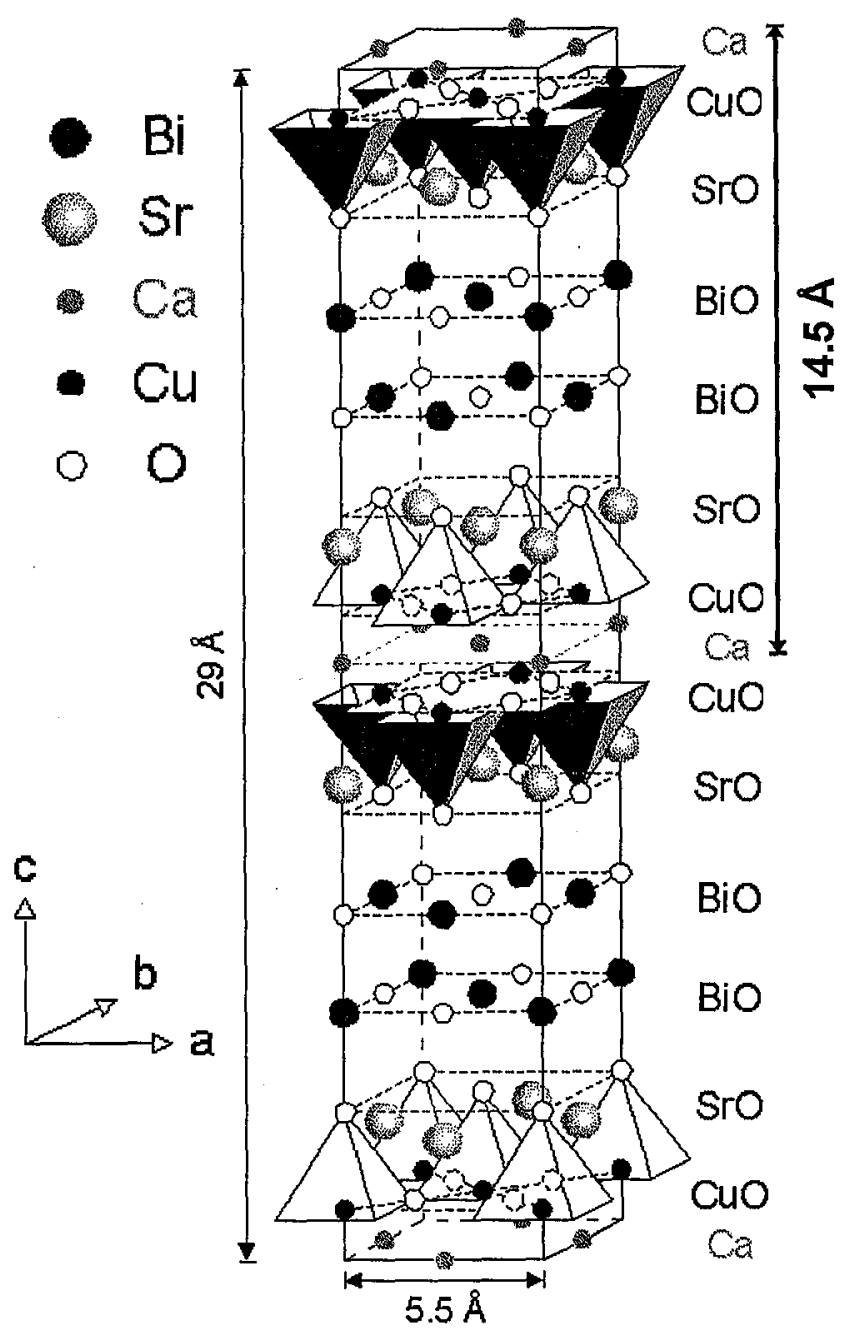

Fig. 6. Crystal structure of Bi-2212 (picture from M. Opel, Walther-Meissner-Institut für Tieftemperaturforschung).

the so-called second-peak effect observed in the $M-H$ loops of magnetization experiments on $\mathrm{Bi}-2212$ single crystals occurs due to this dimensional crossover in the vortex system. Therefore, the decoupling of the vortex lines is driven by the gain in pinning energy when the pancake vortices become free to adjust themselves to point-like pinning sites within each individual $\mathrm{CuO}_{2}$ plane when the magnetic field exceeds $B^{*}$. In this regime the " $\mu$ SR-lineshape" becomes almost symmetric at low temperature giving rise to a strongly reduced "skewness" $\alpha$, cusp-shift and second moment $\left\langle\Delta B^{2}\right\rangle$ [9-11]. Corresponding " $\mu$ SR-lineshapes" for the overdoped and underdoped samples are shown in Fig. 7. It has been shown that such a reduction in the width and the shift of $n(B)$ towards the applied field can be accounted 

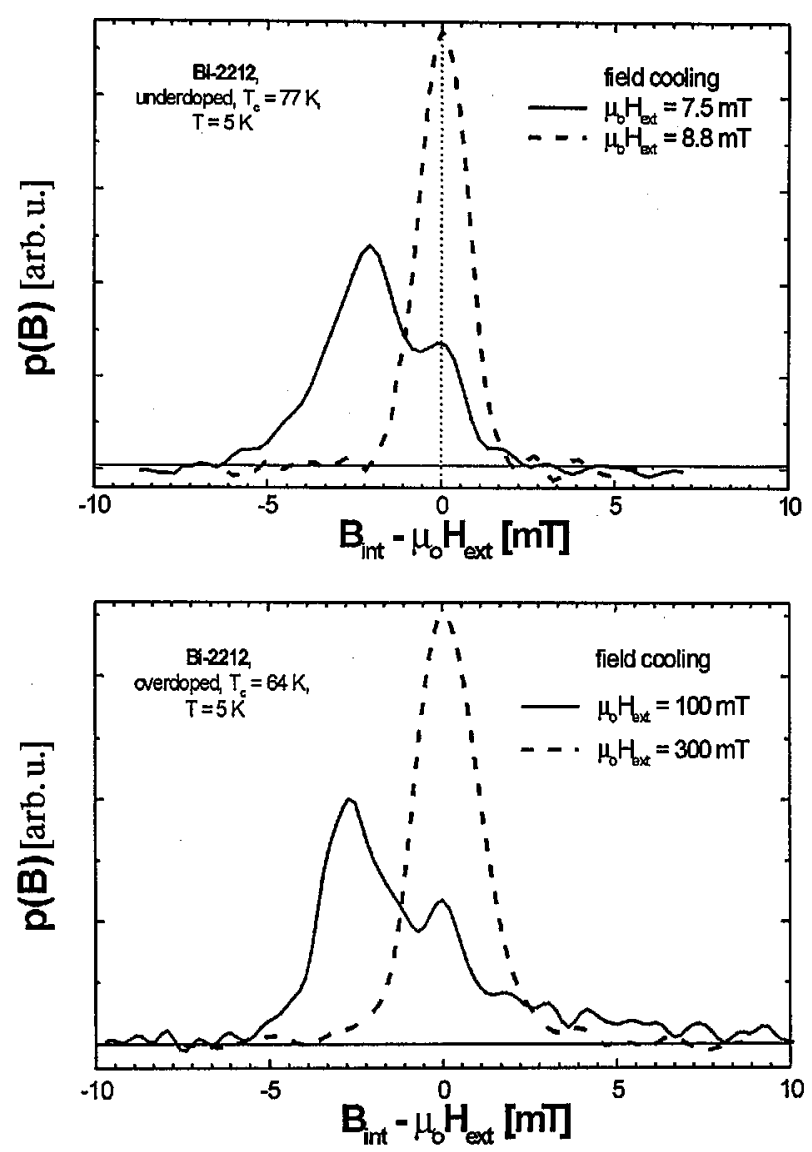

Fig. 7. (Top) The magnetic field distribution at $5 \mathrm{~K}$ determined by TF- $\mu \mathrm{SR}$ for underdoped Bi-2212 crystals in applied fields of $7.5 \mathrm{mT}$ and $8.8 \mathrm{mT}$. The asymmetric field distribution at $7.5 \mathrm{mT}$ show features which are characteristic of a $3 \mathrm{D}$ flux structure. (Bottom) The corresponding results for overdoped crystals in fields of $0.1 \mathrm{~T}$ and $0.3 \mathrm{~T}$.

for by a static model only when the vortices are strongly disordered across the blocking layers while they are still ordered within each block of $\mathrm{CuO}_{2}$ layers. Disorder within the $\mathrm{CuO}_{2}$ planes has the opposite effect, it leads to an increase in the width of $n(B)$. The above described changes in the " $\mu \mathrm{SR}$-lineshape" thus are indicative of a crossover from a $3 \mathrm{D}$ ordered flux line lattice to a quasi-2D vortex structure of pancake vortices where the phase coherence across the neighboring $\mathrm{CuO}_{2}$ layers is destroyed at least on a length scale of $\lambda_{\mathrm{ab}}$.

Figure 8 shows the $\dot{T}$-dependence for $\alpha$ and the cusp-shift which has been obtained for the underdoped set for $H_{\mathrm{ext}}=100 \mathrm{mT}>H^{*} \approx 7.5 \mathrm{mT}$. The most pronounced changes in the " $\mu$ SR-lineshape" occur again in the vicinity of the IL, where $\alpha$ drops suddenly and changes sign from small positive values to larger negative values and where the cusp-shift starts to decrease. Once more a second anomaly is apparent in the $T$-dependence of the " $\mu$ SR-lineshape" which occurs 


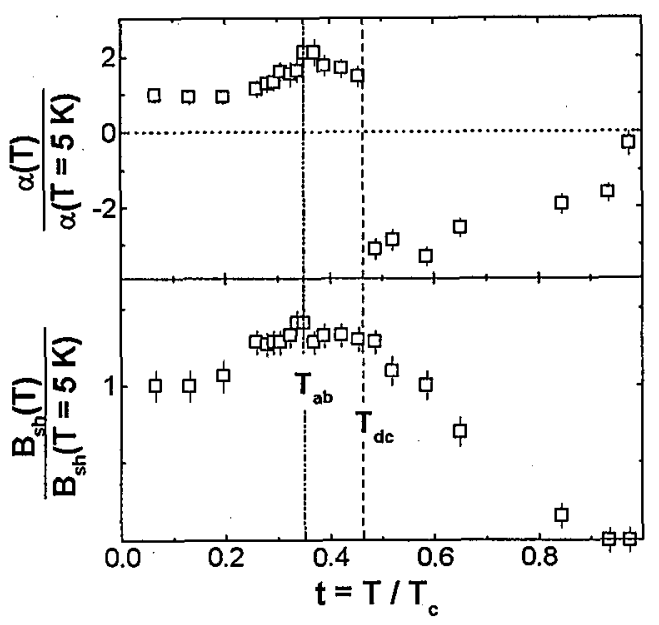

Fig. 8. Data from field-cooled TF $\mu$ SR experiments on the underdoped Bi-2212 single crystal $\left(T_{\mathrm{c}}=77 \mathrm{~K}\right)$ in an applied field of $\mu_{0} H_{\text {ext }}=100 \mathrm{mT}$. Panels are as described in Fig. 4.

in the irreversible regime at a temperature well below the IL. In contrast to the behavior in the FLL state at low magnetic field $H<H^{*}$ (where $\alpha$, the cusp-shift and $\left\langle\Delta B^{2}\right\rangle$ decrease with increasing $T$ ) the "skewness" $\alpha$ now exhibits a small but significant increase and the cusp-shift also becomes somewhat larger. Both effects are indicative of a restoration of the flux-lines as the intra-planar order of the quasi-2D pancake vortex lattice diminishes at $T_{\mathrm{ab}}$. Especially the increase in $\alpha$ signals the formation of vortex-line segments on a length scale along the $c$-axis direction exceeding the in-plane magnetic penetration depth $\lambda_{\mathrm{ab}} \approx 2000 \AA$ [10]. At low $T$ the adjustment to the pinning sites was achieved by suppressing the inter-planar coupling of the individual flux-lines in favor of the persistence of quasi-2D intra-planar order of the pancake vortices. At the in-plane melting transition, however, the intra-planar order is lost and there is no further need for the decoupling of the individual vortex lines in order to obtain a favorable adjustment to the pinning sites. The vortex lines therefore become at least partially restored. We note that the same trend in the temperature dependence of $\alpha$ and the cusp-shift as described above has been observed at a higher applied field of $H_{\text {ext }}=600 \mathrm{mT}$ for the underdoped and the overdoped set of Bi-2212 single crystals and in the temperature dependence of $\alpha$ for an applied field of $H_{\text {ext }}=1 \mathrm{~T}$ for the underdoped set of Bi-2212 single crystal (Fig. 9).

For the above-mentioned fields $H>H^{*}$ we find from pinning experiments that the onset of the vortex depinning occurs in the vicinity of the in-plane melting line, i.e., the vortices are rigidly pinned at temperatures below $T_{\mathrm{ab}}$. This finding supports our previous interpretation [10] that the second-peak effect in the DC-magnetization (anomalous second maximum in the magnetization hysteresis curves [19]) is related to the dimensional crossover from a soft and partially depinned $3 \mathrm{D}$ lattice for $H<H^{*}$ to a rigidly pinned quasi-2D lattice for $H>H^{*}$ (Fig. 10a). The decoupling of the vortex lines and the concomitant enhancement 


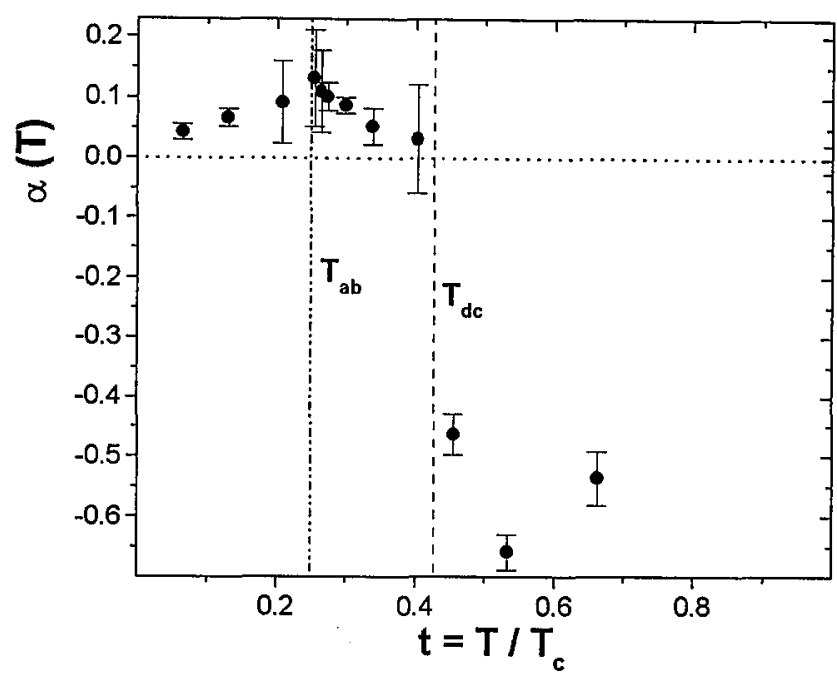

Fig. 9. Temperature dependence of $\alpha$ from field-cooled TF- $\mu$ SR experiments on the underdoped $\mathrm{Bi}-2212$ single crystal $\left(T_{c}=77 \mathrm{~K}\right)$ in an applied field of $\mu_{0} H_{\text {ext }}=1 \mathrm{~T}$.

of the pinning of the quasi-2D pancake lattice increase progressively as the magnetic field is increased above $H^{*}$ until it goes through a maximum right at the melting transition. At even higher magnetic fields in the line-liquid phase the pinning properties decline due to enhanced thermal fluctuations before the magnetic behavior becomes reversible beyond the IL in the pancake liquid phase.

A rather interesting question is how our observation of a two-stage melting transition fits in with the numerous reports of a one-stage melting transition which seems to be firmly established especially for the less strongly anisotropic systems like $\mathrm{Y}_{1} \mathrm{Ba}_{2} \mathrm{Cu}_{3} \mathrm{O}_{7-\delta}(\mathrm{Y}-123)$. It seems likely that the key to the answer lies in the $c$-axis coupling strength which varies strongly amongst the different cuprate high- $T_{\mathrm{c}}$ compounds. In contrast, the condensation energy [20] or the super-fluid density [21] which determines the in-plane vortex-vortex interaction do not vary much amongst the cuprates. In fact, simply by rescaling the $(H, T)$ phase diagram with the critical field of the dimensional crossover $H^{*}$, we obtain a rather unique $(H, T)$ phase diagram from the DC-magnetization data for a wider series of $\mathrm{Bi}-2212$ single crystals (Fig. 10b). A similar trend has been recently reported for a number of different high- $T_{c}$ compounds [22]. This would imply that a two-stage melting process is experimentally observable if the applied magnetic field is comparable to, or larger than $H^{*}$, the field for the dimensional crossover, while a seemingly one-stage melting process occurs when $T_{\mathrm{ab}}$ and $T_{\mathrm{dc}}$ do collapse for $H \ll H^{*}$. In fact, such a trend is even visible from our data on the Bi-2212 crystals for which the $T_{\mathrm{ab}}(H)$ - and $T_{\mathrm{dc}}(H)$-lines seem to merge for $H \ll H^{*}$. For the much less strongly anisotropic Y-123 compound $H^{*}$ should be in excess of $10 \mathrm{~T}$ and accordingly most of the experiments have been done for $H<H^{*}$ where in fact a single-stage transition is observed.

In summary, from muon spin rotation measurements on three sets of overdoped $\left(T_{\mathrm{c}}=64 \mathrm{~K}\right)$, nearly optimized $\left(T_{\mathrm{c}}=90 \mathrm{~K}\right)$, and underdoped $\left(T_{\mathrm{c}}=77 \mathrm{~K}\right)$ 

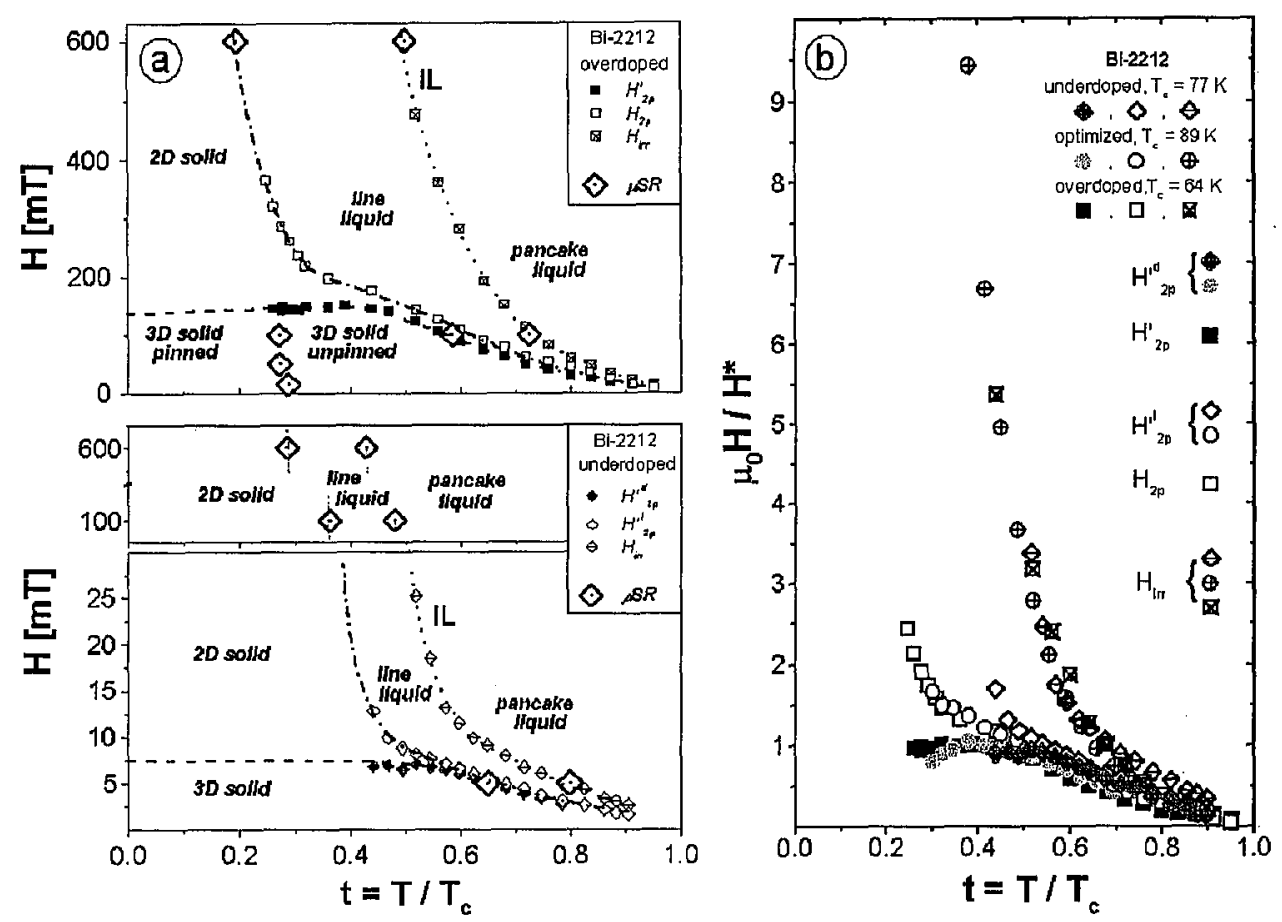

Fig. 10. (a) $(H, T)$-phase diagram constructed from $\mu$ SR and VSM [10] data. Shown are the irreversibility field $H_{\text {irr }}$, the field for the second peak in $M(H), H_{2 \mathrm{p}}$, and for the peak in the corresponding derivative $\mathrm{d} M / \mathrm{d} H, H_{2 \mathrm{p}}^{\prime}$ (index $\mathrm{d}$ for decreasing and $\mathrm{i}$ for increasing field). The lines are guides to the eye. (b) Rescaled VSM data (scaling factor $H^{*}$ ) for different doping states of Bi-2212 single crystals.

$\mathrm{Bi}_{2} \mathrm{Sr}_{2} \mathrm{CaCu}_{2} \mathrm{O}_{8+\delta}$ single crystals, we have obtained evidence for a two-stage melting transition of the vortex matter under equilibrium conditions. Two marked changes in the " $\mu$ SR-lineshape" have been observed and related to transitions in the vortex state. The well-known transition at the higher temperature $T_{\mathrm{dc}}$ coincides with the irreversibility line, as determined from DC-magnetization measurements, and is related to the decoupling of the individual flux-lines which results in a pancake liquid state with reversible magnetic behavior. The second transition of the vortex state occurs at a temperature $T_{\mathrm{ab}}$ in the irreversible regime well below the IL. For both sets of Bi-2212 single crystals this transition is found to coincide with the second-peak effect as seen in the DC-magnetization measurements. We interpret this second transition as the intra-planar melting of the vortex structure which leads to a line-liquid state with irreversible magnetic behavior.

The experiments described herein have been performed at the Paul-Scherrer-Institute, Villigen, Switzerland and the TRIUMF, Vancouver, Canada. We thank these institutions and their support stuff for continuing assistance. The financial support of the German BMFB and the DFG is gratefully acknowledged. 


\section{References}

[1] G. Blatter, M.V. Feigel'man, V.B. Geshkenbein, A.I. Larkin, V.M. Vinokur, Rev. Mod. Phys. 66, 1125 (1994); E.H. Brandt, Rep. Prog. Phys. 58, 1465 (1995).

[2] D.R. Nelson, H.S. Seung, Phys. Rev. B 39, 9153 (1989); E.H. Brandt, Phys. Rev. Lett. 63, 1106 (1989); A. Houghton, R.A. Pelcovits, A. Sudbo, Phys. Rev. B 40, 6763 (1989).

[3] P.L. Gammel, L.F. Schneemeyer, J.V. Waszczak, D.J. Bishop; Phys. Rev. Lett. 61, 1666 (1988).

[4] J.R. Clem, Phys. Rev. B 43, 7837 (1991).

[5] L.I. Glazman, A.E. Koshelev, Phys. Rev. B 43, 2835 (1991); Y.H. Li, S. Teitel, Phys. Rev. B 49, 4136 (1994); S. Ryu, A. Kapitulnik, S. Doniach, Phys. Rev. Lett. 77, 2300 (1996); N.K. Wilkin, H.J. Jensen, Europhys. Lett. 40, 423 (1997); A.K. Nguyen, A. Sudbo, Phys. Rev. B 57, 3123 (1998); S. Ryu, D. Stroud, Phys. Rev. B 57, 14476 (1998); Z. Tesanovic, Phys. Rev. B 59, 6449 (1999).

[6] M.C. Hellerqvist, S. Ryu, L.W. Lombardo, A. Kapitulnik, Physica $C$ 230, 170 (1994); K. Kadowaki, Physica C 263, 164 (1996); C.D. Keener, M.L. Trawick, S.M. Ammirata, S.E. Hebboul, J.C. Garland, Phys. Rev. Lett. 78, 1118 (1997); M.F. Goffman, J.A. Herbsommer, F. de la Cruz, T.W. Li, P.H. Kes, Phys. Rev. B $\mathbf{5 7 ,} 3663$ (1998).

[7] D.T. Fuchs, E. Zeldov, T. Tamegai, S. Ooi, M. Rappaport, H. Shtrikman, Phys. Rev. Lett. 80, 4971 (1998).

[8] R. Cubitt, E.M. Forgan, G. Yang, S.L. Lee, D.Mc K. Paul, H.A. Mook, M. Yethiraj, P.H. Kes, T.W. Li, A.A. Menovsky, Z. Tarnawski, K. Mortensen, Nature 365, 407 (1993).

[9] S.L. Lee, P. Zimmermann, H. Keller, M. Warden, I.M. Savic, R. Schauwecker, D. Zech, R. Cubitt, E.M. Forgan, P.H. Kes, T.W. Li, A.A. Menovsky, Z. Tarnawski, Phys. Rev. Lett. 71, 3862 (1993); S.L. Lee, M. Warden, H. Keller, J.W. Schneider, D. Zech, P. Zimmermann, R. Cubitt, E.M. Forgan, M.T. Wylie, P.H. Kes, T.W. Li, A.A. Menovsky, Z. Tarnawski, Phys. Rev. Lett. 75, 922 (1995); S.L. Lee, C.M. Aegerter, H. Keller, M. Willemin, B. Stäuble-Pümpin, E.M. Forgan, S.H. Lloyd, G. Blatter, R. Cubitt, T.W. Li, P.H. Kes, Phys. Rev. B 55, 5666 (1997); C.M. Aegerter, J. Hofer, I.M. Savic, H. Keller, S.L. Lee, C. Ager, S.H. Lloyd, E.M. Forgan, Phys. Rev. B 57, 1253 (1998).

[10] C. Bernhard, C. Wenger, Ch. Niedermayer, D.M. Pooke, J.L. Tallon, Y. Kotaka, J. Shimoyama, K. Kishio, D.R. Noakes, C.E. Stronach, T. Sembiring, E.J. Ansaldo, Phys. Rev. B 52, R7050 (1995).

[11] T. Blasius, Ch. Niedermayer, J.L. Tallon, D.M. Pooke, A. Golnik, C. Bernhard, Phys. Rev. Lett. 82, 4926 (1999).

[12] N. Motohira, K. Kuwahara, T. Hasegawa, K. Kishio, K. Kitazawa, J. Ceram. Soc. Jpn. 97, 994 (1989).

[13] C.T. Lin et al., private communication.

[14] T.M. Riseman, J.H. Brewer, K.H. Chow, W.N. Hardy, R.F. Kiefl, S.R. Kreitzman, R. Liang, W.A. MacFarlane, P. Mendels, G.D. Morris, J. Rammer, J.W. Schneider, C. Niedermayer, S.L. Lee, Phys. Rev. B 52, 10569 (1995).

[15] T.M. Riseman et al., to be published.

[16] A. Schenck, Muon Spin Rotation Spectroscopy, Adam Hilger, Bristol 1985. 
[17] E.H. Brandt, Phys. Rev. B 37, 2349 (1988); E.H. Brandt, J. Low Temp. Phys. 73, 355 (1988).

[18] J.W. Schneider, S. Schafroth, P.F. Meier, Phys. Rev. B 52, 3790 (1995); E.H. Forgan, M.T. Wylie, S. Lloyd; M.P. Nutley, S.L. Lee, R. Cubitt, C. Aegerter, H. Keller, T.W. Li, Hyperfine Interact. 105, 61 (1997).

[19] V.N. Kopylov, A.E. Koshelev, I.F. Schegolev, T.G. Togonidze, Physica $C$ 170, 291 (1990); T. Tamegai, Y. Iye, I. Oguro, K. Kishio, Physica C 213, 33 (1993).

[20] J.L. Tallon, Phys. Rev. B 58, 5956 (1998).

[21] Y.J. Uemura, A. Keren, L.P. Le, G.M. Luke, W.D. Wu, Y. Kubo, T. Manako, Y. Shimakawa, M. Subramanian, J.L. Kobb, J.T. Markert, Nature (London) 352, 605 (1991).

[22] T. Sasagawa, K. Kishio, Y. Togawa, J. Shimoyama, K. Kitazawa, Phys. Rev. Lett. 80, 4297 (1998). 